\title{
Connective Tissue Disorders in Patients With Thrombotic Thrombocytopenic Purpura: A Retrospective Analysis Using a National Database
}

\author{
Pratibha Sharma $^{\mathrm{a}, \mathrm{d}}$, Aveena Gurung ${ }^{\mathrm{b}}$, Sumit Dahal ${ }^{\mathrm{c}}$
}

\begin{abstract}
Background: Prior reports have shown acquired thrombotic thrombocytopenic purpura (TTP) co-existing with connective tissue disorders (CTD). However, these are mainly limited to case reports and case-series reports, and the patient characteristics and clinical outcomes in these patients are not well known.
\end{abstract}

Methods: We used National Inpatient Sample and Nationwide Inpatient Sample (NIS) database for the years 2009 to 2016 to identify all adult patients with TTP and searched for either the presence or absence of any co-existing CTD. These two cohorts of TTP patients were then compared using statistical methods for baseline patient characteristics and clinical outcomes. The primary outcome of interest was the all-cause in-hospital mortality and the secondary outcomes were in-hospital length of stay, in-hospital total charge and in-hospital complications.

Results: Of the 14,400 cases of TTP diagnosed between 2009 and 2016, nearly 9\% $(\mathrm{n}=1,247)$ had one or more underlying CTD. Patients with TTP were more likely to be young, black, female, with more than one comorbidity and with private insurance if they had an underlying CTD than when they did not have any underlying CTD. There was no difference in regards to the size, location or type of the hospital, or the time taken to initiate plasmapheresis. Patients being managed for TTP had a longer mean length of hospital stay and a greater mean total inpatient stay charge if they had underlying CTD. There was however no difference in the risks of inpatient mortality, acute coronary syndrome, cardiac arrest, acute stroke, need for mechanical ventilation or hemodialysis.

Conclusion: TTP and CTD frequently co-existed and contributed to a longer hospital stay and a greater hospital charge.

Manuscript submitted May 9, 2019, accepted May 27, 2019

aDepartment of Medicine, Maimonides Medical Center, Brooklyn, NY, USA bepartment of Internal Medicine, New York University School of Medicine, Woodhull Medical and Mental Health Center, Brooklyn, NY, USA

${ }^{\mathrm{c} H o s p i t a l i s t ~ S e r v i c e, ~ S t . ~ J o s e p h ~ H o s p i t a l, ~ B a n g o r, ~ M E, ~ U S A ~}$

${ }^{\mathrm{d} C}$ Corresponding Author: Pratibha Sharma, Department of Medicine, Maimonides Medical Center, Brooklyn, NY 11219, USA.

Email: prativasharma20@gmail.com

doi: https://doi.org/10.14740/jocmr3850
Keywords: Thrombotic thrombocytopenic purpura; Connective tissue disorder; Autoimmune disorder

\section{Introduction}

Thrombotic thrombocytopenic purpura (TTP) is a form of thrombotic microangiopathy characterized by microangiopathic hemolytic anemia and thrombocytopenia along with end-organ damage like fever, renal dysfunction and neurological manifestations. It is caused by reduced activity of von Willebrand factor (VWF)-cleaving metalloproteinase called ADAMTS13. It can occur both as a hereditary and as an acquired disorder. Hereditary TTP is related to mutation in the ADAMTS13 gene while acquired TTP is related to the development of autoantibodies against ADAMTS13 protein. Acquired TTP has previously been shown to be associated with various autoimmune disorders [1-4]. With an overlapping autoimmune pathogenetic mechanism, cases of TTP have been reported in the background of or as the presenting feature of different connective tissue disorders (CTD) [5-10]. However, most of the prior literature on the co-existence of TTP and CTD is limited to case reports and case-series reports limited to a single institute experience. Furthermore, the baseline patient characteristics as well as clinical outcomes in patients who develop TTP in the background of underlying CTD are not well known.

\section{Materials and Methods}

We performed retrospective analysis for our study using National Inpatient Sample and Nationwide Inpatient Sample (NIS) database for the years 2009 to 2016 [11]. NIS database is sponsored by the Agency for Healthcare Research and Quality as part of the Healthcare Cost and Utilization Project (HCUP). It is the largest all-payer inpatient care database in the United States, containing annual data on more than 7 million hospital stays unweighted, and estimates more than 35 million hospitalizations nationally when weighted. NIS identifies diseases with International Classification of Diseases, Ninth Revision, Clinical Modification (ICD-9-CM) code for diagnosis for the years 2009 to 2014, with both ICD-9-CM (from January to 
September) and ICD-10-CM (from October to December) codes for the year 2015 and with ICD-10-CM codes for the year 2016.

\section{Study population and data extraction}

We used NIS to identify all patients above 18 years of age with TTP discharged between January 1, 2009 and December 31, 2016 using ICD-9-CM code 446.6 and ICD-10-CM code M31.1. We included only those patients who received therapeutic plasmapheresis (ICD-9-CM procedure code 99.71, and ICD-10-CM procedure codes 6A550Z3 and 6A551Z3) during hospitalization to capture active cases of TTP and improve coding accuracy. The presence of connective tissue diseases - rheumatoid arthritis, systemic lupus erythematosus (SLE), systemic sclerosis, dermatopolymyositis and mixed connective tissue disease - were identified using the respective ICD9-CM and ICD-10-CM codes for diagnosis. The patient characteristics extracted were age, gender, race, comorbidity index score and insurance type. Hospital characteristics of interest were bed size (small, medium or large), location (urban or rural) and teaching status (academic or non-academic). The time duration in days between admission and initiation of plasmapheresis was also extracted.

\section{Outcomes measured}

The primary outcome measured was the all-cause in-hospital mortality of patients who developed TTP. The secondary outcomes of interest were in-hospital length of stay, in-hospital total charge and in-hospital complications of acute coronary syndrome, cardiac arrest, acute stroke, need for mechanical ventilation and need for hemodialysis.

\section{Statistical analysis}

Baseline patient and hospital characteristics were summarized using descriptive statistics and then compared between the cohorts of patients with and without underlying CTD. Categorical variables were compared using the Chi-square test and continuous variables using the Student's $t$-test. Multivariable logistic regression was performed for primary and secondary outcomes of interest. Results were considered statistically significant for P value less than 0.05. STATA/IC 15.1 O Stata Corp LLC was used for all statistical analysis.

Institutional Review Board Approval was not required for our study due to the retrospective nature of the study using a deidentified HCUP database. This study was conducted in compliance with the ethical standards of the responsible institution on human subjects as well as with the Helsinki Declaration.

\section{Results}

There were total 14,400 cases of TTP diagnosed between 2009 and 2016. Almost $9 \%$ of these patients $(n=1,247)$ had one or more underlying connective tissue diseases (Table 1). Patients with TTP were more likely to be female if they had an underlying CTD than when they did not have any underlying CTD $(87.3 \%$ vs. $64 \%, \mathrm{P}<0.01)$. Similarly the proportion of blacks was significantly higher $(48.9 \%$ vs. $39 \%, \mathrm{P}<0.01)$ and the proportion of whites was significantly lower $(34.4 \%$ vs. $47.3 \%, \mathrm{P}<0.01$ ) in patients with TTP and underlying CTD as compared to those with TTP without underlying CTD. The mean age at presentation with TTP was significantly lower in those with underlying CTD as compared to those without CTD (43.87 years vs. 48.64 years, $\mathrm{P}<0.01$ ). Almost half of the patients with TTP and underlying CTD as compared to nearly one-third of those with TTP without underlying CTD (45.9\% vs. $31 \%, \mathrm{P}<0.01)$ had more than one comorbidities. A greater proportion of patients with TTP had private insurance in presence of underlying CTD as compared to those without underlying CTD (53.9\% vs. $39.2 \%, \mathrm{P}<0.01)$. There was no statistically significant difference in the proportion of patients with TTP and underlying CTD and those without underlying CTD in regards to the size, location or type of the hospital where they received their care. Similarly, the mean time taken to initiate plasmapheresis for TTP was similar irrespective of the presence or absence of underlying CTD (2.92 days vs. 2.77 days, $\mathrm{P}=0.5499$ ).

Patients being managed for TTP had a longer mean length of hospital stay and a greater mean total inpatient stay charge if they had underlying CTD (18.2 days vs. 15.3 days and $\$ 76,166$ vs. $\$ 59,358, \mathrm{P}<0.01)$. However, the odds of inpatient mortality in TTP were similar irrespective of the presence or absence of underlying CTD with the adjusted odds ratio (OR) of inpatient mortality in those with underlying CTD as compared to those without CTD being 0.96 (95\% confidence interval $(\mathrm{CI})$ : $0.54-1.70, \mathrm{P}=0.882$ ) (Table 2). Similarly, underlying CTD in the setting of TTP was not associated with higher risks of developing acute coronary syndrome (adjusted OR: 0.84, 95\% CI: $0.43-1.64, \mathrm{P}=0.616$ ), cardiac arrest (adjusted OR: 1.69, 95\% CI: $0.76-3.75, \mathrm{P}=0.201$ ) or acute stroke (adjusted OR: 0.80, 95\% CI: $0.44-1.46, \mathrm{P}$ $=0.468$ ) in comparison to those with TTP without underlying CTD. There was also no significant difference in those with underlying CTD and those without CTD in the setting of TTP in regards to the need for either mechanical ventilation (adjusted OR: 1.24, 95\% CI: $0.76-2.04, \mathrm{P}=0.389$ ) or hemodialysis (adjusted OR: 1.39, 95\% CI: $0.97-2.00, \mathrm{P}=0.075$ ) during their hospitalization.

\section{Discussion}

TTP is a rare but potentially life-threatening multisystemic disorder $[12,13]$. It has classically been described by the pentad of microangiopathic hemolytic anemia, thrombocytopenia, fever, renal dysfunction and neurological manifestations [14]. However, early and regular use of plasmapheresis has significantly dropped mortality and improved overall clinical outcomes [15, 16]. As such, early clinical diagnosis of TTP without the classical pentad of features is important. Microangiopathic hemo- 
Table 1. Baseline Characteristics of Patients With Thrombotic Thrombocytopenic Purpura

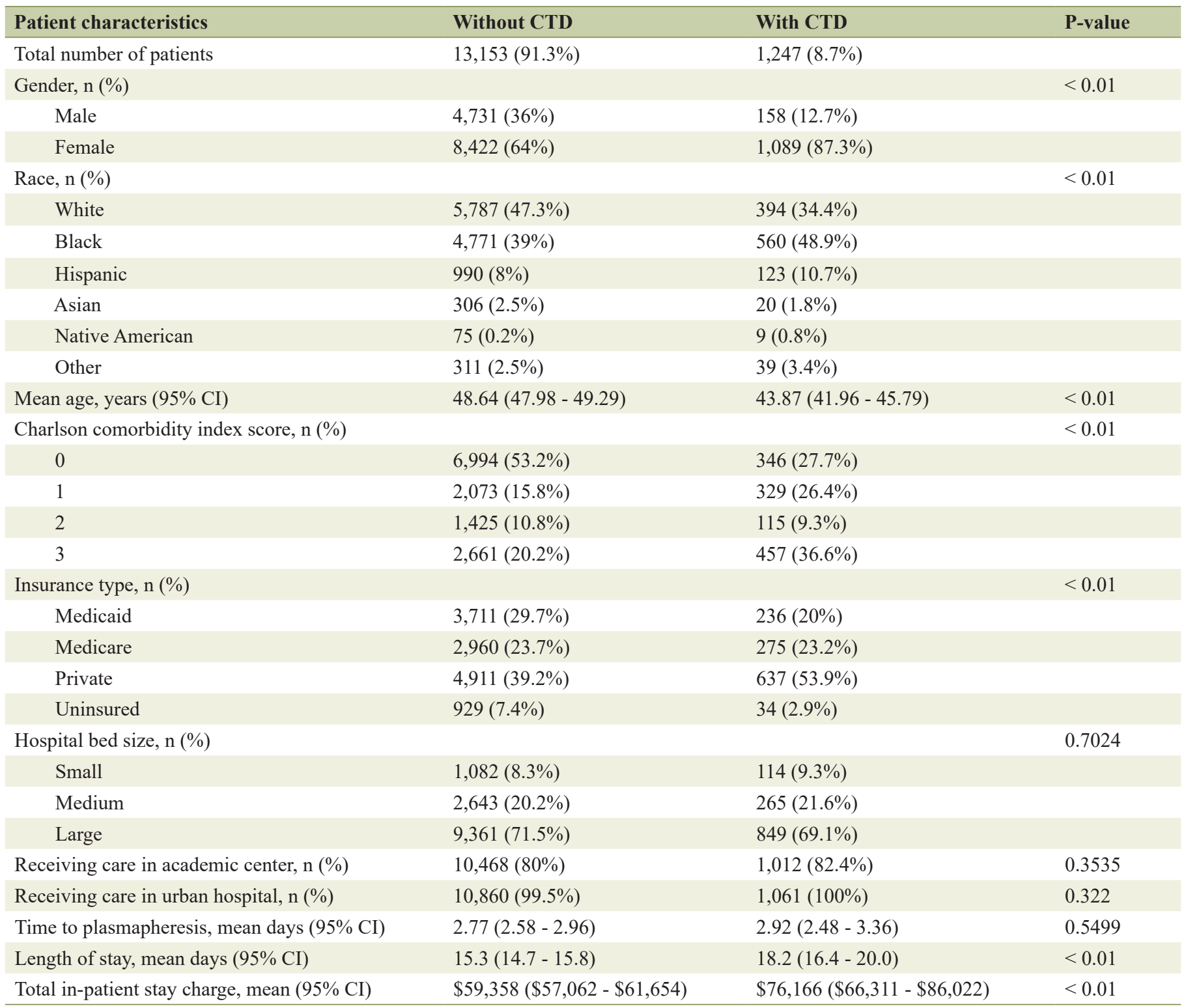

CTD: connective tissue disorders; $\mathrm{Cl}$ : confidence interval.

Table 2. Clinical Outcomes of Thrombotic Thrombocytopenic Purpura in Patients With Connective Tissue Disorders as Compared to Those Without Connective Tissue Disorder

\begin{tabular}{llll}
\hline & Adjusted odds ratio & $\mathbf{9 5 \%}$ confidence interval & P-value \\
\hline $\begin{array}{l}\text { Primary outcome } \\
\quad \text { Mortality }\end{array}$ & 0.96 & $0.54-1.70$ & 0.882 \\
$\begin{array}{l}\text { Secondary outcomes } \\
\quad \text { Acute coronary syndrome }\end{array}$ & 0.84 & $0.43-1.64$ & 0.616 \\
$\quad$ Cardiac arrest & 1.69 & $0.76-3.75$ & 0.201 \\
$\quad$ Acute stroke & 0.80 & $0.44-1.46$ & 0.468 \\
$\quad$ Need for mechanical ventilation & 1.24 & $0.76-2.04$ & 0.389 \\
$\quad$ Need for hemodialysis & 1.39 & $0.97-2.00$ & 0.075 \\
\hline
\end{tabular}


lytic anemia and thrombocytopenia in the absence of alternative explanation is usually sufficient for clinical diagnosis and initiation of live saving plasma exchange transfusions.

Reduced activity of VWF-cleaving ADAMTS13 protease is central in the pathogenesis of TTP, and can be secondary to inherited mutation in the ADAMTS13 gene or the development of autoantibodies against ADAMTS13 protein. VWF is a glycoprotein secreted by endothelial cells in response to endothelial injury. It is initially secreted as unusually large multimers that are subsequently cleaved by ADAMTS13. In the setting of ADAMTS13 deficiency, there is an excess of unusually large VWF multimers (UL-VWFM) in circulation potentiating platelet aggregation and adhesion leading to widespread arteriolar thrombosis and luminal stenosis. Development of anti-ADAMTS13 autoantibodies can either be idiopathic or secondary to conditions like drugs, malignancy, pregnancy and CTD.

The underlying pathophysiological basis for TTP occurring in CTDs is, however, not entirely clear and possibly multifactorial. In a study by Matsuyama et al, patients with CTD and TTP were shown to have significantly lower average plasma levels of ADAMTS13 activity compared to normal population [17]. However, they tended to have higher average plasma levels of ADAMTS13 activity and a significantly lower proportion with severe deficiency of ADAMTS13 as compared to those with idiopathic TTP (17\% vs. $70 \%)$. Besides the inhibitory antibodies against ADAMTS13, role of immunemediated endothelial injury and non-inhibitory antibodies that could trigger TTP by promoting ADAMTS13 clearance from the circulation or by altering its cell surface expression have been proposed $[6,18,19]$. Decreased ADAMTS13 activity and elevated VWF levels have been observed in patients with CTD $[6,20,21]$. Furthermore, patients with CTD tend to develop vasculitis that subsequently generate high shear stress besides impeding the binding of ADAMTS13 for efficient cleavage of UL-VWFM [22]. These factors accelerate platelet clumping in the presence of high plasma levels of VWF leading to thrombotic microangiopathies including TTP.

TTP can be seen in the background of CTD or as the initial presentation of CTD [5-9]. It has been reported in $2 \%$ of patients with SLE [17]. However, this is mainly reflective of patients with known SLE and may be an underestimation as patients presenting with TTP are rarely worked up for presence of CTD. Furthermore, a variety of secondary autoimmune disorders may develop subsequently at various timeframes after TTP $[1,4,23]$. This makes estimating the correct association of TTP and CTD highly challenging. In our study, nearly $9 \%$ of the patients with TTP had one or more underlying CTD. This is consistent to prior analysis by Roriz et al in which $10 \%$ of patients with TTP had a medical history of autoimmune disease [1]. Furthermore, they reported a cumulative frequency of pre-existing, concomitant or subsequent autoimmune disease to be $21.5 \%$ in patients with TTP. The presence of either antiSSA antibodies or anti-dsDNA antibodies at the time of TTP diagnosis was associated with a marked risk of subsequent autoimmune disorder during follow-up [1].

Timely identification of TTP in the setting of CTD can be challenging due to the overlapping clinical features. Each of the clinical feature of TTP- hemolytic anemia, thrombocytope- nia, renal failure, neurological features and fever- can also be seen with CTD, and may wrongly be attributed to a CTD flare $[24,25]$. Presence of schistocytes on peripheral smear, a negative Coombs test, normal complement levels and a severely deficient ADAMTS13 activity favor the diagnosis of TTP [26]. The coexistence of CTD and TTP has been reported to be more common in young black women $[6,27,28]$. This is consistent with the findings of our study where patients with TTP and CTD had a lower mean age as well as a higher proportion of blacks and females than the TTP patients without any CTD. SLE disease activity index score, coexisting nephritis and lymphopenia were previously shown to be independent risk factors for TTP in the setting of SLE $[17,28]$. Though rare, the association of CTD and TTP is potentially lethal and warrants early diagnosis and aggressive management with plasma exchange and immunosuppression. Rituximab is increasingly used for refractory cases [29-31].

There have been conflicting reports in regards to the clinical outcomes of TTP in the presence of CTD. Some previous studies had reported higher mortality with worse overall prognosis in patients with TTP and CTD than with TTP alone [32, 33]. Two small case series previously reported mortality rates of $46-50 \%$ in TTP in the presence of CTD, and was associated with presence of concomitant infections and a delay in initiation of plasmapheresis $[17,26]$. In our study, presence of underlying CTD resulted in a longer hospital stay and a greater in-hospital cost for patients with TTP. However, there was no difference in mortality or other clinical outcomes. Such lack of effect of the presence of CTD on the clinical outcomes of TTP is consistent with some previous studies $[1,6]$. There was no association with mortality, relapse or exacerbation. Similar clinical outcomes in patients with TTP and CTD or TTP alone in our study may be related to the comparable average day before plasmapheresis was initiated in the two groups. Such early initiation of plasmapheresis may also be the reason for lower overall mortality in our cohort of patients with TTP and CTD as compared to similar cohorts in some other studies. Interestingly, a retrospective study by Merayo-Chalico et al showed a lower mortality in TTP with CTD than in TTP alone [28]. And this was true despite no differences regarding the time to plasmapheresis after diagnosis. The same study, in lieu of the findings of our current study, showed a longer hospitalization stay without any difference in either the need for mechanical ventilation or hemodialysis in TTP patients with CTD as compared to those without CTD. The longer hospital stay in these patients was possibly related to their significantly higher rate of infection owing to their greater immunosuppression.

There are several limitations to our study. Besides the inherent limitation of a retrospective study design, our study is based on discharge-level patient data with limited clinical information. Furthermore, this study utilizes a de-identified administrative database and is prone to coding errors that could not be individually verified. And with our case definition for TTP consisting of patients who had undergone plasmapheresis treatment during the same hospitalization, the reported clinical outcome circumvents the diagnostic and therapeutic dilemma of initiating plasmapheresis. However, our study utilized a large sample size available through a national database and, 
to our knowledge, is the largest study to look into the co-existence of TTP and CTD.

\section{Acknowledgments}

This study utilized the HCUP NIS database. The interpretation of the data is the sole responsibility of the authors. The authors acknowledge the efforts of Agency for Healthcare Research and Quality and its HCUP Data Partners in the creation of the HCUP NIS database. The list of HCUP Data Partners is available on https://www.hcup-us.ahrq.gov/db/hcupdatapartners.jsp.

\section{Financial Disclosure}

None.

\section{Conflict of Interest}

None.

\section{Informed Consent}

Not applicable.

\section{Author Contributions}

PS, AG and SD developed the concept for the study. SD designed and conducted the study. PS, AG and SD were involved with data analysis and interpretation of the results. SD drafted the manuscript. PS and AG reviewed and critically revised the manuscript. All the authors approved the final version of the manuscript.

\section{References}

1. Roriz M, Landais M, Desprez J, Barbet C, Azoulay E, Galicier L, Wynckel A, et al. Risk factors for autoimmune diseases development after thrombotic thrombocytopenic purpura. Medicine (Baltimore). 2015;94(42):e1598.

2. Pandey RK, Dahal S, Fadlalla K, Bhagat S, Bhattarai B. Acquired thrombotic thrombocytopenic purpura in a patient with pernicious anemia. Case Rep Hematol. 2017;2017:1923607.

3. Kosaka M, Takahashi N, Saitoh H, Masai R, Ito M, Sato $\mathrm{R}$, Wakui H, et al. Thrombotic thrombocytopenic purpura with severe ADAMTS-13 deficiency in a patient with antiphospholipid antibodies and Charcot-Marie-Tooth disease. Intern Med. 2011;50(5):487-493.

4. John ML, Scharrer I. Autoimmune disorders in patients with idiopathic thrombotic thrombocytopenic purpura. Hamostaseologie. 2012;32(Suppl 1):S86-89.

5. Pan YX, Wang HY, Shang YT, Wang XZ, Wang Q, Tian
JM, Zhang JY, et al. [Clinical analysis of 12 cases of systemic lupus erythematosus associated with thrombotic thrombocytopenic purpura]. Zhongguo Shi Yan Xue Ye Xue Za Zhi. 2017;25(4):1147-1150.

6. Matsuyama T, Kuwana M, Matsumoto M, Isonishi A, Inokuma S, Fujimura Y. Heterogeneous pathogenic processes of thrombotic microangiopathies in patients with connective tissue diseases. Thromb Haemost. 2009;102(2):371378.

7. Viner M, Murakhovskaya I. A rare combination of thrombotic thrombocytopenic purpura and antiphospholipid syndrome. Blood Coagul Fibrinolysis. 2017;28(5):411415.

8. Suzuki E, Kanno T, Asano T, Tsutsumi A, Kobayashi H, Watanabe H, Ohira H. Two cases of mixed connective tissue disease complicated with thrombotic thrombocytopenic purpura. Fukushima J Med Sci. 2013;59(1):49-55.

9. Bamidele OF, Akintayo RO, Bojuwoye MO, Alabi TO, Akintayo FC, Bamidele OV. Thrombotic thrombocytopenic purpura as the first presentation in systemic lupus erythematosus. Reumatologia. 2018;56(4):268-270.

10. Toumeh A, Josh N, Narwal R, Assaly R. Refractory thrombotic thrombocytopenic purpura associated with primary Sjogren syndrome treated with rituximab: a case report. Am J Ther. 2014;21(2):e56-60.

11. HCUP Nationwide and National Inpatient Sample (NIS). Healthcare Cost and Utilization Project (HCUP). 20092016, Agency for Healthcare Research and Quality, Rockville, Md.

12. Reese JA, Muthurajah DS, Kremer Hovinga JA, Vesely SK, Terrell DR, George JN. Children and adults with thrombotic thrombocytopenic purpura associated with severe, acquired Adamts 13 deficiency: comparison of incidence, demographic and clinical features. Pediatr Blood Cancer. 2013;60(10):1676-1682.

13. Crawley JT, Scully MA. Thrombotic thrombocytopenic purpura: basic pathophysiology and therapeutic strategies. Hematology Am Soc Hematol Educ Program. 2013;2013:292-299.

14. Amorosi EL, Ultmann JE. Thrombotic thrombocytopenic purpura: report of 16 cases and review of the literature. Medicine. 1966;45(2):139-160.

15. Rock GA, Shumak KH, Buskard NA, Blanchette VS, Kelton JG, Nair RC, Spasoff RA. Comparison of plasma exchange with plasma infusion in the treatment of thrombotic thrombocytopenic purpura. Canadian Apheresis Study Group. N Engl J Med. 1991;325(6):393-397.

16. von Baeyer H. Plasmapheresis in thrombotic microangiopathy-associated syndromes: review of outcome data derived from clinical trials and open studies. Ther Apher. 2002;6(4):320-328.

17. Kwok SK, Ju JH, Cho CS, Kim HY, Park SH. Thrombotic thrombocytopenic purpura in systemic lupus erythematosus: risk factors and clinical outcome: a single centre study. Lupus. 2009;18(1):16-21.

18. Mariani M, Cairo A, Palla R, Lotta LA, Consonni D, Rovati $\mathrm{A}$, Trisolini $\mathrm{S}$, et al. B and T lymphocytes in acquired thrombotic thrombocytopenic purpura during disease remission. Thromb Res. 2011;128(6):590-592. 
19. Gugliesi F, Bawadekar M, De Andrea M, Dell'Oste V, Caneparo V, Tincani A, Gariglio M, et al. Nuclear DNA sensor IFI16 as circulating protein in autoimmune diseases is a signal of damage that impairs endothelial cells through high-affinity membrane binding. PLoS One. 2013;8(5):e63045.

20. Habe K, Wada H, Matsumoto T, Ohishi K, Ikejiri M, Tsuda K, Kondo M, et al. Plasma ADAMTS13, von Willebrand Factor (VWF), and VWF propeptide profiles in patients with connective tissue diseases and antiphospholipid syndrome. Clin Appl Thromb Hemost. 2017;23(6):622-630.

21. Pagano L, Marra R, Paoletti S, Afa G, Storti S, Garcovich A, Bizzi B. Factor VIII complex in progressive systemic sclerosis. Clin Exp Rheumatol. 1986;4(4):319-321.

22. Moake JL. von Willebrand factor, ADAMTS-13, and thrombotic thrombocytopenic purpura. Semin Hematol. 2004;41(1):4-14.

23. Dimopoulou D, Dimosiari A, Mandala E, Dimitroulas T, Garyfallos A. Autoimmune thrombotic thrombocytopenic purpura: two rare cases associated with juvenile idiopathic arthritis and multiple sclerosis. Front Med (Lausanne). 2017;4:89.

24. Dold S, Singh R, Sarwar H, Menon Y, Candia L, Espinoza LR. Frequency of microangiopathic hemolytic anemia in patients with systemic lupus erythematosus exacerbation: Distinction from thrombotic thrombocytopenic purpura, prognosis, and outcome. Arthritis Rheum. 2005;53(6):982-985.

25. Manadan AM, Harris C, Schwartz MM, Block JA. The frequency of thrombotic thrombocytopenic purpura in patients with systemic lupus erythematosus undergoing kidney biopsy. J Rheumatol. 2003;30(6):1227-1230.

26. Shah AA, Higgins JP, Chakravarty EF. Thrombotic microangiopathic hemolytic anemia in a patient with SLE: diagnostic difficulties. Nat Clin Pract Rheumatol.
2007;3(6):357-362.

27. Terrell DR, Vesely SK, Kremer Hovinga JA, Lammle B, George JN. Different disparities of gender and race among the thrombotic thrombocytopenic purpura and hemolyticuremic syndromes. Am J Hematol. 2010;85(11):844-847.

28. Merayo-Chalico J, Demichelis-Gomez R, Rajme-Lopez S, Aparicio-Vera L, Barrera-Vargas A, Alcocer-Varela J, Gomez-Martin D. Risk factors and clinical profile of thrombotic thrombocytopenic purpura in systemic lupus erythematosus patients. Is this a distinctive clinical entity in the thrombotic microangiopathy spectrum?: a case control study. Thromb Res. 2014;134(5):1020-1027.

29. Limal N, Cacoub P, Sene D, Guichard I, Piette JC. Rituximab for the treatment of thrombotic thrombocytopenic purpura in systemic lupus erythematosus. Lupus. 2008;17(1):69-71.

30. Hundae A, Peskoe S, Grimsley E, Patel S. Rituximab therapy for refractory thrombotic thrombocytopenic purpura and autoimmune-mediated thrombocytopenia in systemic lupus erythematosus. South Med J. 2008;101(9):943-944.

31. Hie M, Gay J, Galicier L, Provot F, Presne C, Poullin $\mathrm{P}$, Bonmarchand $\mathrm{G}$, et al. Preemptive rituximab infusions after remission efficiently prevent relapses in acquired thrombotic thrombocytopenic purpura. Blood. 2014;124(2):204-210.

32. Jiang H, An X, Li Y, Sun Y, Shen G, Tu Y, Tao J. Clinical features and prognostic factors of thrombotic thrombocytopenic purpura associated with systemic lupus erythematosus: a literature review of 105 cases from 1999 to 2011. Clin Rheumatol. 2014;33(3):419-427.

33. Letchumanan P, Ng HJ, Lee LH, Thumboo J. A comparison of thrombotic thrombocytopenic purpura in an inception cohort of patients with and without systemic lupus erythematosus. Rheumatology (Oxford). 2009;48(4):399403. 